\title{
Wallis-et-Futuna (1942-1961) ou comment le fait migratoire transforma le protectorat en TOM
}

\section{Frédéric Angleviel}

\section{(2) OpenEdition \\ 1 Journals}

Édition électronique

URL : http://journals.openedition.org/jso/541

DOI : $10.4000 /$ jso. 541

ISSN : $1760-7256$

Éditeur

Société des océanistes

Édition imprimée

Date de publication : 1 décembre 2006

Pagination : 61-76

ISSN : 0300-953x

\section{Référence électronique}

Frédéric Angleviel, «Wallis-et-Futuna (1942-1961) ou comment le fait migratoire transforma le protectorat en TOM », Journal de la Société des Océanistes [En ligne], 122-123 | Année 2006, mis en ligne le 01 décembre 2009, consulté le 01 mai 2019. URL : http://journals.openedition.org/jso/541 DOI : $10.4000 /$ jso. 541

(c) Tous droits réservés 


\section{Wallis-et-Futuna (1942-1961) ou comment le fait migratoire transforma le protectorat en TOM}

par

Frédéric ANGLEVIEL*

\section{RÉSUMÉ}

À partir de nouvelles sources archivistiques ${ }^{l}$, nous présenterons la fin du protectorat de Wallis-et-Futuna, marquée par l'impact durable de la présence américaine. Après le départ des Américains en 1946, les Wallisiens et les Futuniens surent éviter les errements du cargo cult tout en intégrant de nouvelles pratiques relationnelles à leur organisation sociale très hiérarchisée. Cette période vit l'effondrement de la monoculture commerciale de l'archipel, l'instauration d'une migration de travail et l'apparition du fait politique. Le pouvoir coutumier, doublé depuis 1837 par l'influence spirituelle du clergé catholique et confronté depuis 1888 à l'influence temporelle de la résidence de France, accepta en effet en 1959 la mise en place d'institutions politiques occidentales. Le fait que le statut de 1961 soit aujourd'hui encore inchangé s'explique par une répartition rigidifiée des cinq pouvoirs en présence (chefferie, clergé, administration, négoce et politique) destinée initialement à les équilibrer.

Mots-CLÉs : Wallis-et-Futuna, protectorat, ÉtatsUnis, pouvoir, migration, politique.

\begin{abstract}
Thanks to new sources found in the archives, we will deal here with the final years of the protectorate in Wallis and Futuna, a period marked by the lasting impact of American presence. After their departure in 1946. Wallisians and Futunians knew how to avoid the erring ways of the cargo cult while integrating new relational practices to their highly stratified social organization. This transitional period witnessed the downfall of the commercial monoculture of the archipelago, the setting up of a migration scheme for workers and the emergence of political consciousness. Customary power - duplicated as it was by the spiritual influence of the Catholic clergy since 1837, and confronted to the temporal influence of the French resident since 1888 indeed accepted the setting up of Western political institutions in 1959. If the 1961 statute remains unchanged to this day, the explanation lies in a highly rigid distribution of the five powers involved (customary, religious, administrative, commercial and finally political), initially aimed at keeping them in balance.
\end{abstract}

KeYwords : Wallis and Futuna, protectorate, United States, power, migration, politic.

1. Notre contribution s'appuie sur deux missions de recherche aux archives de l'évêché de Wallis-et-Futuna ainsi que sur l'étude des archives du Protectorat à la Délégation des îles Wallis-et-Futuna en Nouvelle-Calédonie, jointes à la très riche thèse de géographie de Jean-Claude Roux (1991).

* Professeur en histoire contemporaine, université de la Nouvelle-Calédonie, angleviel@univ-nc.nc 
Le protectorat s'articule en trois périodes que l'on peut nommer : l'isolement (1888-1918); la mise en place d'un véritable pouvoir administratif (1919-1941) ; l'ouverture sur le monde extérieur (1942-1961). Nous étudierons ici la période 1942-1961, avec le départ des Américains et ses conséquences immédiates (Angleviel, 1997), l'évolution politique des deux archipels qui en découla, ainsi que les difficultés économiques réelles de l'après-guerre. Le temps des Amelika est aujourd'hui encore synonyme d'abondance et de mutations rapides. Mais quel fut réellement son impact sur ces deux microcosmes? Comment les Wallisiens et les Futuniens purent continuer durablement à bénéficier des biens matériels qu'ils avaient appris à apprécier ?

\section{Les crises de l'après-guerre}

Suite à la folle abondance initiée par la présence américaine, les années de l'immédiate après-guerre connurent des crises avant qu'intervienne une longue dépression économique.

\section{La période américaine ou le choc des cultures}

Depuis 1940, le résident Vrignaud, épaulé par $\mathrm{M}^{\mathrm{gr}}$ Poncet $^{2}$ et le nouveau roi de Wallis, Leone Manikitoga, étaient restés fidèles à l'État de Vichy, préférant la «famine » à la « farine ». Le 27 mai 1942, après dix-sept mois d'un isolement maritime total, l'aviso FFL Chevreuil débarque un nouveau résident, le capitaine médecin Mattéi (Regnault, 1997 : 3). Dans les semaines qui suivent, les Américains installent une base aérienne sur l'île.

De 1942 à juin 1944, Wallis reçoit en moyenne quatre mille GI à haut pouvoir d'achat. Entre leurs menues dépenses, l'emploi de plus de blanchisseuses et les nombreux chantiers commandités par l'armée us, l'île connaît un enrichissement sans précédent. Géographiquement, la répartition des offres américaines s'avère équilibrée car la principale piste d'aviation est au nord à Hihifo, le colonel commandant les troupes est à Afala, près de Lano, et leur base navale se situe dans le sud, à Gahi. Du reste, les trois aumôniers catholiques qui se succèdent entre 1942 et 1944 dans l'île célèbrent tous les dimanches la messe dans les trois districts. À cela s'ajoute toute la panoplie de guerre : un matériel impressionnant est débarqué, qui laissa une empreinte durable après guerre: maisons préfabriquées, citernes, véhicules automobiles en grand nombre et même les premiers bulldozers de l'île. Enfin, des liaisons régulières par bateau et par avion sont mises en place avec les Samoa. Il en résulte une extraordinaire prospérité économique à la fois inattendue, brève et sans lendemain. Une véritable folie de consommation s'abat sur l'île malgré les efforts de réglementation de la résidence. Un officier des renseignements américains écrit ainsi en 1944 :

« The retail end of the establishment has prospered mightily, since buying power is unbelievabily high. Shipments of merchandise and food-stuffs desired can be brought in only sporadically, and are quickly exhausted by purchase in a few days. Principal favorites; cloth for lava-lava; flour; sugar; rice; canned meat. Sale of alcoholic beverages is forbidden. $»^{3}$ (Anonyme, 1944 : 8)

Cette dernière interdiction explique la création de distilleries artisanales clandestines par les soldats américains. $\mathrm{M}^{\mathrm{gr}}$ Poncet note en 1945 :

«Kava papalagi : est-ce qu'on en fabrique encore à Mua ? Y a-t-il des indigènes qui en boivent ? $»^{4}$

Sur le plan humain, la première conséquence de cette arrivée massive de soldats est une sérieuse épidémie de grippe, qui entraîne le décès de vingr-sept personnes en juin 1942. Peu après, un petit incident tranche avec la monotonie habituelle. Le père Simler, alsacien, étant né dans cette province au temps de la domination allemande, est considéré comme persona non grata. Finalement, il part pour Futuna par le Cap des Palmes le 20 août 1942 et un prêtre wallisien est muté en échange à Wallis.

Sur l'ensemble du séjour, quelques rixes ont lieu entre des soldats et des jeunes gens, entrầnant le décès de trois Wallisiens. Par ailleurs, $\mathbf{M}^{\mathrm{gr}}$ Poncet signale que les Américains ordonnent l'envoi de trois cents Wallisiens en NouvelleCalédonie. Quelques-uns n'étant pas volontaires

2. Lettre de A. Poncet, SM, évêque de Basilinopolis à monsieur le résident, Lano, 29 avril 1942 (Archives de Lano, Carton VII, chemise a2) : « Je m'efforcerai toujours de soutenir votre action pour maintenir Wallis dans son devoir de fidélité à la France et à son illustre chef, le maréchal [...] Ce problème du ravitaillement me paraît plus que jamais un des points noirs de notre situation ».

3. «Les commerces de détail ont énormément prospéré du fait que le pouvoir d'achat est incroyablement élevé. Les cargaisons de marchandises et de produits alimentaires arrivent de manière sporadique et les achats les épuisent en quelques jours. Les produits les plus recherchés sont : le tissu pour les lava (paréo), la farine, le riz, le corn-beef. La vente de boissons alcoolisées est interdite ».

4. Compte rendu de la réunion des missionnaires, 27 décembre 1945 (feuille volante dans le cahier Diaire 1943-1949, archives de Lano, carton DIV). 
ou laissant derrière eux une famille, l'évêque intervient et finalement c'est un contingent de cent-soixante-neuf travailleurs, dont quarantehuit Futuniens, qui prend l'avion pour Nouméa.

La société wallisienne est strictement hiérarchisée et les aînés ou les familles nobles ('aliki) détiennent prestige et pouvoir. Seuls les cadets acceptent d'aller travailler pour les Amelika, ce qui leur permet aussi d'échapper à leurs nombreuses obligations coutumières. Il en découle un enrichissement soudain des dépendants, qui remet partiellement en cause la hiérarchie établie. Ceci amène l'administration coloniale à réévaluer les indemnités des chefs coutumiers de mille pour cent en 1943. De même, une part notable de l'autorité de ces mêmes chefs reposant sur l'imposition de lourdes amendes, leur principal moyen de pression s'affaiblit, les roturiers (tua) pouvant désormais facilement les payer en dollars. Il est à signaler que la résidence a repris ce mode de fonctionnement local et que, dès 1943, les amendes administratives, portées pour la plupart à 50 dollars, ont rapporté 110000 francs.

Parallèlement, l'impôt de capitation est doublé à Wallis sans problème et les blanchisseuses payent une « patente »; à cela s'ajoute surtout l'application des droits de douane à de nombreux produits américains. Tous ces faits expliquent une explosion des recettes alors que les dépenses sont quasiment nulles. Le budget du protectorat devient alors très largement excédentaire et le gouverneur de Nouvelle-Calédonie réaffecte aux Nouvelles-Hébrides la subvention annuelle attribuée par le gouvernement de la France libre 5 . La résidence a à cœur d'accompagner les actions américaines et c'est ainsi qu'une route de 12 mètres de large est créée au centre de l'île. De même, lors du départ du gros des troupes alliées, si la majorité du matériel est réembarquée ou, par ordre supérieur, jeté à la mer, la résidence et la mission obtiennent de conserver qui des citernes, qui des planches ou du mobilier. La récupération est systématique et étalée sur plusieurs années. Le résident, par exemple, en 1947 réclame à la mission le chaland métallique que celle-ci a récupéré, afin de le couler au bout du wharf dans le but de créer un appontement correct.
Des changements psychologiques et culturels ont résulté de cette brutale rencontre entre une population préservée et le monde occidental. L'argent facile en est un des éléments, mais tout aussi importante est la découverte d'une puissance matérielle insoupçonnée ou la matérialisation de nouveaux rapports humains. Dans le même temps, cette recherche effrénée de biens matériels a parfois débouché sur des pratiques critiquables, comme le vol ou la prostitution. Pour le moins, des actes mole katolico («pas catholique ») et un certain relâchement des mœurs sont constatés par la mission. À l'inverse de la résidence et de la mission, les Américains considèrent les Wallisiennes comme particulièrement farouche :

«Native women apparently keep their chastity because of fear if they are young, of the French nun in charge of their convent school; if they are married, of their husbands. $)^{6}$ (Anonyme, $1944: 23-24$ )

Parallèlement des liens d'amitié se tissèrent et $\mathrm{M}^{\mathrm{gr}}$ Poncet note :

"The Americans at last realised that those who they thought first to be primitive savages had in fact a way of greeting and entertaining foreigners, above all those of their own creed, showing the most affectionate feeling [...] Friendship being so established, those coming from a great and opulent continent, seeing the absolute poverty of their new friends for war conditions had stopped the regular supply of the island - were so glad to help them in many ways $[\ldots] »^{7}$

Tout aussi important fut le contact avec des soldats de couleur et Robert Aldrich (1993 : 28) estime à juste titre que :

«The impact of seeing black American soldiers working on an equal basis with white soldiers is not to be underestimated. $»^{8}$

Dès mars 1944 il ne reste plus que trois cents GI et, à partir de juin 1944 l'île ne comprend plus que douze soldats américains, ramenant les Wallisiens face à eux-mêmes. En 1945, un navire vient même des Samoa américaines pour chercher les restes mortuaires de la dizaine de soldats morts accidentellement durant leur séjour à Wallis. Brusquement, la situation (re)devient pré-

5. Rapport de Fourcade, directeur de cabinet du gouverneur, au gouverneur de la Nouvelle-Calédonie, Nouméa, septembre 1943 (Délégation de Wallis-et-Futuna en Nouvelle-Calédonie [DWF], carton correspondance).

6. «Apparemment, les femmes indigènes font preuve de chasteté par peur, si elles sont jeunes, des nonnes en charge des couvents ; si elles sont mariées, de leurs maris. » L'auteur cite trois cas de prostitution notoire et il donne la liste des seize femmes soignées pour des blennorragies.

7. Lettre de Mgr Poncet to his excellency Bishop O'Hara, Lano, 30th march 1943, 6 pages (archives de Lano, carton DVII, chemise A1).

8. «Il ne faut pas sous-estimer l'impact que représenta pour les Wallisiens le fait de voir les soldats noirs américains travailler sur une base égale avec les soldats de race blanche » 
caire, alors que les pouvoirs traditionnels sortent affaiblis de la tourmente : la mission est confrontée à une baisse de la moralité ; le contrôle de la coutume devient un enjeu économique ; l'administration coloniale est à rebâtir. Il est vrai que, dès 1943, l'influence américaine est devenue prédominante dans l'île. Un officier américain participe ainsi à toutes les réunions du fono royal alors que des projets de longue durée sont proposés à l'attention de la chefferie comme l'instauration d'une base d'hydravions de la PanAm. Tout cela et l'antagonisme traditionnel entre autorités civiles et militaires ont créé à la fin de la guerre un malaise entre les représentants des autorités françaises et américaines.

Un épiphénomène révélateur intervient en 1946. Selon les témoignages du résident (Charbonnier, 1980) et de l'évêque, le dernier chef du dernier détachement us, le lieutenant Zincheck, a eu une attitude inamicale envers les autorités civiles. Or, une manifestation comportant à sa tête ce même lieutenant et des ministres coutumiers déchus en 1944 apporte à la résidence le 25 mars 1946 une pétition demandant «l'annexion de Wallis aux Etats-Unis et le départ des Français ». Stupeur des trois pouvoirs traditionnels en place et saine réaction du résident qui retient le petit peloton calédonien de rétablir immédiatement l'ordre, évitant un affrontement direct franco-américain. Le $\mathrm{D}^{\mathrm{r}}$ Charbonnier obtient finalement le retrait des soldats américains et les excuses du lieutenant Zincheck. L'affaire a eu des répercussions coutumières jusqu'au 9 avril, date du départ définitif du contingent américain, mais l'envol dès le 30 mars du lieutenant Zincheck achève de convaincre de sa défaite l'active minorité pro-américaine. Les conséquences sont multiples. Sur le plan factuel, le Lavelua Leone songe à abdiquer le 26 mars mais le résident lui confirme son soutien. La mission enlève aux ofisa, officiers du Tiers Ordre ${ }^{9}$ qui s'étaient compromis, leurs responsabilités politico-religieuses.

Parallèlement, la fin du rêve américain débouche sur une brutale décompression économique. Le gouverneur Parisot écrit ainsi en 1948 :

« La population indigène de ces îles se trouve dans le dénuement le plus complet, manquant des objets les plus courants et les plus indispensables. Il en résulte un mécontentement, dont les répercussions sur le plan politique se feraient de plus en plus sentir, si nous nous montrions incapable d'améliorer dans un bref délai, cette situation déplorable au double point de vue national et simplement humain. ${ }^{10}$

\section{Une mono-économie en crise}

Durant ces quatre années, on note un abandon des plantations de cocotiers car la main-d'œuvre se trouve sur les chantiers américains et l'absence de cargos empêche toute exportation. Les cocos sont donc perdus ou servent à nourrir les porcs. Les cultures vivrières connaissent un désintérêt analogue, du fait que les rations alimentaires américaines sont distribuées généreusement avec un grand gaspillage institutionnel.

Parallèlement, autant les Wallisiens apprécient les boîtes de conserve, autant les Américains aiment la viande fraîche. C'est ainsi que le petit cheptel de l'île est sur le point de disparaître et que dix têtes de bétail seulement ont survécu. Cochons et poules sont victimes de véritables hécatombes et, une fois l'euphorie retombée, la chefferie doit mettre un tabou sur leur consommation afin de permettre une reconstitution du stock. Même le lagon a été mis à mal, du fait d'une pêche abusive et de la pratique - pourtant interdite - de la pêche à la dynamite. Aussi, lors de la raréfaction puis de la disparition de la manne américaine, les Wallisiens acceptent difficilement le retour à une économie quasi autarcique. Le médecin commandant Chomet note ainsi :

« Si la soudure au point de vue cultures vivrières est un peu difficile $[\ldots]$ on exagère de suite et on parle de famine. $»^{11}$

Enfin, un dangereux parasite du cocotier, l'Oryctes rhinoceros, déjà présent dans l'île avant la Deuxième Guerre mondiale, a proliféré en l'absence de mesures préventives. Aussi, après une période de quasi-abandon, les Wallisiens se remettent au travail sur leurs plantations. La résidence trouve que la remise en état des cocoteraies a été réalisée avec beaucoup de négligence et $\mathrm{M}^{\mathrm{gr}}$ Poncet considère que cette même administration doit contrôler beaucoup plus les Wallisiens, afin de les obliger à développer leurs cultures vivrières et la production de coprah. Quoi qu'il en soit, la production retrouve dès 1948 le niveau d'avant-guerre, mais les conditions du marché des oléagineux et les aspirations des Wallisiens ont changé.

9. Le Tiers Ordre est la quatrième branche de la congrégation mariste : il s'agit de laïcs qui font des vœux simples pour des durées limitées.

10. Lettre du gouverneur Parisot à monsieur le ministre de la France d'Outre-Mer, Nouméa, 24 janvier 1948 (DWF, carton archives divers). Voir aussi le rapport suivant : Le gouverneur de la Nouvelle-Calédonie à monsieur le ministre de la France d'Outre-Mer, Nouméa, 23 juillet 1947 (archives de la Nouvelle-Calédonie, AT I07W).

11. Rapport du médecin-commandant Chomet au haut-Commissaire de la France dans le Pacifique, 21 novembre 1947 (DWF, carton correspondance). 


\section{Une permanence de l'isolement pour Futuna}

Depuis toujours privée de lagon, Futuna n'offre aux navires que l'aléatoire rade foraine de Leava, vite dangereuse, ce qui amène cette île à rester repliée sur elle-même (Moyse-Faurie, 2002). De même, son relief montagneux permet la perpétuation des clivages. Ces réalités géographiques expliquent une fois encore son évolution différente de celle de Wallis lors de la Deuxième Guerre mondiale.

Durant toute la guerre Futuna a été une fois de plus isolée. Toutes les liaisons commerciales sont coupées, seuls quelques trop rares navires de guerre et hydravions militaires frayent dans les parages. C'est ainsi que le Cap des Palmes y fait une rapide escale en août 1942 ou qu'un hydravion chasseur de sous-marins se rend pour trois jours à Futuna le 22 juin 1943. Il en ramène quarante-cinq Futuniens, qui sont les premiers à prendre l'avion et à s'engager hors de leur île. Ils passent tout le temps de la guerre à travailler sur le sol calédonien et, bien qu'aucune étude ne porte sur leur retour, il semble qu'ils soient presque tous rentrés dès que les liaisons sont redevenues normales en 1947. Si l'on en croit un des ministres coutumiers de Sigave, le Sa'atula Soane Kaikilekofe, que j'avais interviewé à ce sujet à Futuna en 1982, ce sont les mêmes familles qui ont envoyé ensuite les premiers engagés, tant militaires que civils, en NouvelleCalédonie.

Le 10 novembre $1942, M^{\text {gr }}$ Poncet se rend à Futuna sur un navire américain et malgré sa promesse le général Price est incapable de le ramener avant le 21 décembre, et ceci sur un minuscule navire. Seulement quatre autres touchées ont lieu durant le reste de la guerre : un aller-retour à Wallis pour permettre à l'évêque de rencontrer ses fidèles quelques jours, deux navires en campagne, un contre-torpilleur et un sous-marin. En ce qui concerne une éventuelle liaison par hydravion, un essai est fait le 26 juin 1943 afin de permettre au résident de faire pour une journée acte de présence à Futuna. En raison de la difficulté pour amerrir près d'une île montagneuse dépourvue de lagon et du total désintérêt militaire pour cet archipel, les autorités américaines refusent toute autre tentative. À part cela, de trop rares hydravions ont lâché du courrier au-dessus du siège de la station missionnaire, à Sausau. Un navire de commerce français militarisé, l'Oiseau des îles, conduit le $1^{\text {er }}$ août 1943 le résident Mattéi, le roi Leone, un missionnaire et vingt-cinq Futuniens pour un pèlerinage au tom- beau de Saint-Pierre Chanel. Selon l'usage, ces Futuniens arrivés à 'Uvea au fil des ans ont patiemment attendu qu'un navire accepte de les rapatrier.

Bien encadrés par leurs deux chefferies et la mission, les Futuniens ont continué à faire du coprah et, du fait de la pénurie de matières premières, ils en ont tiré un bon prix. Aussi, à la fin du conflit mondial, ont-ils du numéraire, mais la seule maison commerciale, Burns et Philp, n'a plus aucune marchandise depuis longtemps, ce qui l'a du reste conduit à distribuer plus de deux millions de francs CFP en bons de caisse. Le père Cantala, supérieur de la mission et délégué du résident note :

«Depuis 1940, date de la fermeture du magasin de Burns Philip (sic), la population s'est habillée à l'aide, soit de dons qui lui ont été faits de Wallis, soit de vêtements obtenus à bord de navires de passage par le moyen d'échange avec des tapas indigènes, soit surtout de sacs à coprah usagés, cédés à prix d'argent par les représentants de $\mathrm{B}$ and $\mathrm{P} . »^{12}$

Par ailleurs, il regrette que le chemin du tour de l'île soit à l'abandon et que le wharf soit très abîmé, tout en se félicitant de la pureté des mœurs de ses fidèles. En 1948, le père Cantala, qui devra lui-même attendre 1956 pour pouvoir se faire opérer de deux hydrocèles, signale une épidémie de typhoïde consécutive au passage du Polynésien.

Durant toute cette période, Futuna, surnommée «l'enfant perdu » par Louis-Antoine de Bougainville, continue à vivre dans l'isolement. C'est ainsi que les places sur les bateaux étant vendues à l'avance à Nouméa, les Futuniens n'arrivent pas à avoir un contingent de places suffisant. Puis, lorsque l'avion arrive à Wallis, les passagers se détournent de la voie maritime, ce qui paradoxalement accentue l'isolement de Futuna. En 1963, Michel Panoff constatait encore le quasi-abandon de Futuna. Il précise même :

«Les innovations en la matière sont surtout le fait des habitants de Sigave, leurs voisins d'Alo ne se risquant guère à franchir la frontière pour solliciter un arbitrage qui les exposerait à de possibles représailles ; mais le jeu des forces économiques aura bientôt raison de leurs appréhensions en même temps qu'il arrachera le « royaume » à son isolement. » (Panoff, 1963 : 154)

\section{Crises cumulées des autorités traditionnelles}

La présence américaine ayant représenté un véritable électrochoc, l'équilibre des pou-

12. Rapport politico-économico-social du père Cantala, délégué de Futuna, au résident, Nouméa, 13 mai 1948 (DWF, chemise religion). 
voirs a été rompu et de nouvelles aspirations se manifestent.

\section{Recrudescence des révolutions de palais à Wallis et à Futuna}

À Wallis, trois rois se succèdent de 1945 à 1950. Si le Lavelua Leone Manikitoga a survécu à la crise de 1946, il tombe après quelques manifestations de force en avril 1947. D'après $\mathbf{M}^{\text {gr }}$ Poncet, le résident Chomet prend sur lui de désigner le 9 juin un ministre coutumier originaire de Mua, Pelenato Fuluhea, jugé énergique et francophile. De nouveaux différents coutumiers interviennent en avril 1950, relayant les problèmes sociaux de l'époque : autoritarisme de certains fonctionnaires; clivage des générations dans le clergé local ; retour de Wallisiens sensibilisés aux idées "syndicales ». Le mouvement est parti de Hihifo au sujet du travail non rémunéré et $\mathrm{M}^{\mathrm{gr}}$ Poncet essaise sans succès « d'arrêter ce qui paraissait un mouvement révolutionnaire ». Le roi démissionne le 11 avril. Le surlendemain, les ministres élisent Emmanuel Brial, de famille royale par sa mère. L'administration et la mission ne désirant pas qu'un citoyen français, catholique peu pratiquant, gérant de la principale affaire commerciale, devienne roi de Wallis, le résident l'amène à refuser cette élection. On recourt donc le 17 avril à l'élection d'un roi de transition, Kapeliele Tufele, âgé de 70 ans. Il affirme pourtant rapidement son autorité et dès le mois d'août, le résident fait part à l'évêque d'un premier conflit diplomatique :

« J'ai été averti ce matin qu'à la fête de Hahake, je ne recevrai pas la première coupe de kava. Après en avoir discuté avec le roi et kivalu, j'ai accepté que la première coupe soit offerte au roi mais me soit tout aussitôt offerte par le roi pour la boire. Ce n'est pas que cette solution me soit agréable, mais devant l'intransigeance du roi j'ai dû trouver une solution amiable. Le puleaga va en discuter. Je saurai la décision prise ce soir [...]. Il est donc possible que je n'assiste pas à la fête [...] souhaitant - si cela vous paraît possible de vous voir conformer votre position à la mienne. ${ }^{13}$

En 1953, le résident, qui est en conflit permanent avec le roi, tente pour la troisième fois le 16 novembre, cette fois-ci avec succès, de l'obliger à abdiquer. Un nouvel imbroglio successoral se met en place et finalement c'est madame veuve Julien Brial qui au retour de Nouméa le 22 décembre est élue le jour même, devenant la reine
Aloisia. Le père Mansion remarque que la maison Ballande, amie de toujours des missions et en affaire avec la famille Brial, est intervenue en faveur de cette solution auprès du hautcommissaire de la France dans l'océan Pacifique tout en poussant Benjamin Brial a plus de modération ${ }^{14}$.

On entre dans une période d'instabilité et de vives tensions, où la monarchie essaye d'accompagner les mutations en cours tout en écartant la tutelle jugée trop pesante des résidents. Or, en raison de sa fermeté, que d'aucuns appellent autoritarisme, la reine est bientôt en butte à une forte opposition interne. De plus, d'après les résidents, elle n'a pas le soutien de la mission. À l'inverse, si l'on en croit $\mathrm{M}^{\mathrm{gr}}$ Poncet, elle aurait été en butte aux attaques de la résidence, qui lui reproche de ne pas vouloir céder définitivement un terrain à Hihifo et de refuser les limites imposées par l'avenant de 1910 au protectorat. En novembre 1957, la crise devient constitutionnelle car Aloisia, mise en minorité par le conseil royal, refuse de démissionner. Il en résulte que le district de Mua fait quasiment sécession. Finalement, elle démissionne le 12 septembre 1958 et après des négociations serrées, Tomasi Kulimoetoke est élu le 12 mars 1959. Un mois après, certains anciens ministres veulent élire un autre roi et le Lavelua les oblige à se disperser. Puis, il envoie de jeunes guerriers confisquer à chacun des intéressés un porc de bonne taille. Cet acte d'autorité, la recherche d'une certaine stabilité et la mise en place d'un nouveau statut valorisant le titulaire de la chefferie (indemnité, honneurs renouvelés, voyages) expliquent que Wallis est aujourd'hui encore dirigée par le même Lavelua.

À Futuna, la situation diffère de celle de Wallis compte tenu de l'isolement de l'île, de sa division en deux royaumes et de l'influence forte du père Cantala. Dans un rapport au résident, il ne cache pas ses réticences devant les modifications prônées par l'administration :

« Il est permis de se demander quels avantages réels, apportera à la paix et au bonheur des indigènes, l'accroissement de leurs besoins par une connaissance plus grande par exemple de la langue française. »

Là aussi, la modernité est représentée par la famille Brial, Benjamin Brial y installant en 1950 un négoce. Bientôt, les deux chefferies, soutenues officieusement par le père Cantala, mettent un interdit (tapu) sur la vente de coprah à ce com-

13. Lettre du résident à monseigneur, Mata-Utu, 14 août 1950 (archives de Lano, carton DVII, chemise A2).

14. «En gros voici comment se sont passées les choses. Le Dr a reçu un blâme de son patron. Brial aussi. Aloisia a été reconnue et on lui demandait de s'entendre avec le résident «qui a toute la confiance du Haut-Commissaire ». Situation ennuyeuse pour le résident, qui aurait voulu une solution plus énergique ». Lettre du père Mansion à $M^{g r}$ Poncet, Lano, 15 février 1954 (archives de Lano, carton DVI, chemise C2). 
merçant et il doit revenir temporairement à Wallis. Les deux royaumes changent régulièrement de roi et le père Cantala constate en 1952 la destitution du Tamolevai, notant que si la raison morale invoquée par les chefs n'a pas été acceptée par le résident :

«[Celui-ci] a soulevé lui-même d'autres griefs contre Tamole, griefs fondés surtout sur des plaintes ou rapports qui lui avaient été faits soit directement soit par lettre. Il a toutefois donné l'ordre de ne rien changer à l'ordre actuel jusqu'au jour où il pourra venir lui-même régler la chose ici. Mais les chefs d'ici ne veulent rien entendre et s'obstinent à ne pas vouloir reprendre un contact avec Tamole [...]. Il n'y a plus de punitions infligées, alors les vols se multiplient, les offices religieux ne sont plus suivis, les sacrements sont de moins en moins fréquentés. Je suis moi-même fatigué d'avoir sans cesse à me débattre avec ces difficultés, aussi je ne vous cache pas mon désir de voir ma fonction de délégué confiée à quelqu'un de qualifié pour la remplir, malgré tous les ennuis qui ne manqueront pas d'en résulter. $»^{15}$

Le père Cantala ajoute dans un second courrier en octobre 1952 :

« Notre population a pris l'habitude de faire ce qu'elle voulait, n'étant pas gênée par la crainte des punitions. Je ne sais pas encore comment elle réagira lorsque le gouvernement indigène sera rétabli. »

La même année, deux batailles rangées opposent les villages d'Alo. Le TuiAgaifo Lolesio est prêt à se démettre, mais la population attend la permission réglementaire du résident pour réunir les familles 'aliki concernées. Malgré les dires des résidents, les missionnaires n'usent point après-guerre de moyens coercitifs pour maintenir la pratique religieuse à Futuna, préférant comme le père Cantala laisser « au bon Dieu le soin de les faire fructifier [nos recommandations] en temps opportun ». En revanche, ces mêmes missionnaires regrettent que la pratique diminue rapidement lorsque la chefferie ne veille plus à son respect. Les informations sur Alo s'avèrent beaucoup plus réduites car le seul pôle d'activité de l'île, le village-centre administratif-port de Leava, se trouve à Sigave. De plus, la mission place traditionnellement les pères autochtones en priorité dans la paroisse d'Alo, plus «pure » et plus dure.

Une autre « affaire » intervient en 1955 lorsque Sileno, un Futunien ayant longtemps vécu à
Fidji, prend de l'autorité dans le royaume de Sigave. Il est sur le point d'être arrêté abusivement par le résident lorsque ses partisans rossent le garde du protectorat. L'arrivée du navire de guerre calédonien affecté à la patrouille annuelle change le rapport de forces et Sileno est emprisonné. Il voulait remplacer le roi Keletaona qui l'avait dénoncé au résident comme rebelle à son autorité. Le Keletaona est néanmoins déposé quelque temps plus tard pour un membre de la famille Tamole. Celui-ci, à son tour, est destitué en janvier 1957 au profit d'un autre Keletaona. Le père Simler constate que les raisons de ces changements perpétuels sont difficiles à définir, mais qu'elles se réfèrent toujours à des querelles anciennes. Suite au conflit né du référendum, le roi Keletaona est remplacé en 1960 par un partisan de la territorialisation, Tamole. En 1957 le père Cantala quitte l'île alors que l'arrivée de deux gendarmes fait entrer Futuna dans l'ère de la normalisation administrative. En 1959, un premier délégué civil y est affecté, suivi en 1960 d'un premier médecin. À Alo, le roi Maituku est remplacé en janvier 1958 par Setefano Tuikalepa. Le père Simler note que :

« [il était] soi-disant la cause du vent et du soleil, mais au fond il était trop sévère pour les punitions et faisait tuer trop de cochons ; il était très bon mais il s'est laissé gagner par Sepe Brial paraît-il. Où est la vérité ? ${ }^{16}$

\section{Crise ou recentrage de la mission}

Durant toute la période concernée, l'ensemble de la population affirme sa religiosité et la meilleure définition de la situation religieuse de l'archipel est rapportée par $\mathrm{M}^{\mathrm{gr}}$ Poncet :

« An officer said once to a native priests : "What's that? All catholic here seem to be catholic: I see them all going to Mass on sundays: men, women and children. It is a good country!" $\gg{ }^{17}$

Depuis l'arrivée du roi David en 1933, la résidence a inversé l'équilibre des pouvoirs, tout au moins dans l'île de Wallis. En effet, l'absence pendant huit ans d'un Lavelua a habitué les chefs coutumiers à prendre en compte les ordres de l'administration du protectorat. Le résident Vrignaud, pourtant proche de la mission, écrit en 1940 suite à un démêlé avec la chefferie :

15. Lettre du père Cantala à monseigneur, Sigave, 26 août 1952 (archives de Lano, carton DV, chemise G) ; Lettre du père Cantala à monseigneur, Sigave, 27 octobre 1952 (archives de Lano, carton DV, chemise G).

16. Lettre du père Simler à monseigneur, Sigave, $1^{\mathrm{er}}$ février 1958 (archives de Lano, carton DV, chemise G).

17. «Un officier eut l'occasion de dire à un prêtre indigène : "Qu'est ce que cela ? Tous les catholiques de l'île semblent être catholiques. Je les vois tous aller à la messe chaque dimanche, hommes, femmes et enfants. C'est un pays remarquable'. " Lettre de $M^{g r}$ Poncet to his excellency Bishop O'Hara, Lano, 30th march 1943, 6 p., p. 5 (archives de Lano, carton DVII, chemise AI). 
«Monseigneur, je vous rapporte ces faits qui vous montrent dans quelle situation fausse et pénible, les ordres directement donnés par vos pères indigènes mettent les chefs indigènes et les habitants eux-mêmes. Dans une précédente conversation, j'ai eu l'honneur de vous dire que chaque fois que vous désireriez qu'un travail soit exécuté au bénéfice de la mission, je me ferais un plaisir et un devoir de l'inclure dans les programmes de travaux d'intérêt général et de le faire exécuter, mais que je n'admettrais jamais une dualité de commandement vis-à-vis des chefs et des indigènes. $»^{18}$

Puis, la mise en place, en 1942, d'une petite, mais indépendante, force de police assoit définitivement le pouvoir de la résidence. De même, la Deuxième Guerre mondiale rendant nécessaire une politique d'union sacrée et impliquant un strict respect des autorités, la mission prend l'habitude de ne plus s'impliquer directement sur le plan temporel. Aussi, après-guerre, un nouvel état d'esprit empreint de neutralisme politique se développe au sein du clergé. Le père Mansion écrit ainsi en 1954 :

« Je me tiens prudemment à l'écart, tâchant d'être bien avec tout le monde, n'allant proposer mes conseils à personne, n'en donnant qu'à qui vient m'en demander. Ça fait à peu près quinze jours que je n'ai pas vu le Résident. Je souffle un peu car pendant le mois de janvier je n'ai jamais été plus d'une demi-semaine sans être honoré de sa visite. Il venait ou bien m'apporter à traduire un télégramme envoyé par l'autre camp, ou donner des nouvelles. Toujours très aimable et serviable. ${ }^{19}$

Le principal point de friction de la mission avec l'administration reste l'enseignement. Adapté aux réalités locales, mais insuffisant pour permettre l'entrée de Wallis-et-Futuna dans le monde moderne, il fallait le modifier. La mission et la résidence trouvent rapidement un terrain d'entente, qui évite à l'une l'apparition d'un système laïque concurrent, et à l'autre l'improvisation d'un enseignement public coûteux. Le but de tous les résidents est alors d'arriver à l'alignement rapide de "l'acquis scolaire des élèves uvéens sur celui des écoliers indigènes de la Nouvelle-Calédonie, pour permettre à quelques-uns d'entre eux d'aborder les cours de l'école des cadres » (Angleviel, 2003). De fait les progrès seront lents, les enseignants de la mission n'étant pas toujours qualifiés et l'administration dégageant des moyens insuffisants, soit seulement $5 \%$ du budget global. $\mathrm{M}^{\mathrm{gr}}$ Poncet note en 1960 :
« $1^{\circ}$ Le terrible isolement des îles Wallis-et-Futuna ; $2^{\circ}$ le peu de moyens mis dans le passé, par le gouvernement, à la disposition du seul enseignement chargé de l'éducation des enfants (une subvention annuelle inférieure à 80 francs par élève). Par contre il faut remarquer les efforts tentés dernièrement pour l'enseignement du français et la formation des cadres. »

En 1961 dans un rapport-témoignage, $\mathrm{M}^{\mathrm{gr}}$ Poncet rappelle que le nombre d'élèves scolarisables est pratiquement le même que celui des enfants scolarisés et sans doute avec exagération que « l'enseignement est donné en français dans toutes les écoles depuis $1949 »$. Une nouvelle phase s'ouvre, dont le Futunien Apeleto Likuvalu dit en 1975 :

« Avec le changement de statut en 1961 et la relance de l'émigration sous une forme plus moderne, l'école s'oriente alors vers une francisation intégrale et vers la formation de « l'homme industriel » [...]. Ce qui caractérise cette période et l'enseignement, c'est la scolarisation obligatoire des enfants, l'utilisation du français comme seule langue autorisée [...]. Les choses changent également dans le domaine du corps enseignant : on exige maintenant la possession du diplôme. Les classes deviennent mixtes [...]. » (Likuvalu, 1975 : 39-41)

Dans les autres domaines, il faut bien constater que la mission, riche de son histoire et de son inculturation, possède une véritable influence morale dans le protectorat. Parallèlement, elle assure depuis toujours les missions temporelles habituelles de l'Église : soin et réconfort des malades, enseignement des enfants, conseils politiques et moraux aux notables. Si la résidence tient parfois un discours anticlérical pour sa hiérarchie, elle a généralement à cœur de s'entendre sur le terrain avec la mission, qui est un élément clé de la vie locale. Progressivement, la résidence met en place les infrastructures médicales et éducatives modernes souhaitables. La mission, consciente de l'enjeu, facilite cette transition du moment qu'il ne s'agit pas d'une transposition servile de modèles européens, craignant l'importation des idées laïques. C'est ainsi qu'en 1949 le conseil d'administration de l'évêché décide à l'unanimité que :

«[...] le terrain de Falikata, à Mua, propriété de la Mission, soit mis à la disposition de l'administration gratuitement pour l'érection d'un dispensaire et d'une maternité pour le district de Mua. Une sœur SMSM, résidant au couvent de l'école des Sœurs de Mua, sera chargée du soin des malades et pensionnaires de cet établissement, sous la direction du Médecin de

18. Lettre du résident à monseigneur, Wallis, 9 septembre 1940 (archives de Lano, carton DVII, chemise AI).

19. Lettre du père Mansion à $M^{g r}$ Poncet, Lano, 15 février 1954 (archives de Lano, carton DVI chemise c2). 
l'Administration, le terrain de Falikata restant, du reste, propriété de la mission ».

Une constante apparaît : la mission est favorable à tout ce qui peut améliorer la vie de ses fidèles, du moment que la morale chrétienne est sauvegardée. Sur le plan des idées, cela peut avoir été considéré comme un frein par certains résidents. En effet, les comptes rendus du conseil du vicariat, de la réunion annuelle des missionnaires ou du presbytérium de l'évêché font bien ressortir la surveillance morale étroite que le clergé exerce sur le peuple de Dieu : les manquements aux rites catholiques sont critiqués, les actes considérés comme immoraux tel le fait de s'approcher d'une fille dans son sommeil (moetolo) sont condamnés (Pechberty, $2004: 259)$ et un encadrement à la vie quotidienne est proposé, parfois même imposé. Mais les conditions ont changé et $\mathrm{M}^{\mathrm{gr}}$ Poncet freine le zèle de son clergé, les jeunes prêtres autochtones étant réputés plus sévères que le personnel missionnaire. Il doit ainsi limiter leur propension à construire des églises ou des chapelles.

Concrètement, la mission se révèle un filtre bienfaisant. En effet, on ne peut étudier l'impact de la religion catholique ou celui du clergé de la même manière en Europe ou en Océanie. Ici, la religion (lotu) limite les effets négatifs de l'irruption occidentale, propose en tenant compte des susceptibilités locales de nouveaux modèles de vie à ses fidèles. Le nouveau lotu fait donc disparaître, au profit de l'ensemble des Wallisiens et des Futuniens, des désagréments aussi importants que la guerre et son cortège de destructions, l'amputation rituelle de phalanges lors de décès importants ou encore le pouvoir discrétionnaire des nobles ('aliki) sur les roturiers (tua). Et même si les sermons s'appuient plus souvent sur le Dieu vengeur de l'Ancien Testament que sur le Christ rédempteur des Évangiles, l'impact de la mission s'affirme globalement positif.

En 1951, le résident Folie-Déjardins constate :

« La société wallisienne semble actuellement sans cadres. L'autorité de la mission s'affaiblit sans que l'autorité civile ait encore bien assis la sienne. Le gouvernement lui-même n'exerce qu'un pouvoir limité. Il sait qu'il est à la merci du premier mécontent : les gouvernements, ici, tiennent rarement plus de deux ans. $»^{20}$

Cette assertion est à moduler car la hiérarchie religieuse est à même de faire entendre son point de vue lorsque cela lui semble nécessaire. Dans son ouvrage-témoignage, $\mathrm{M}^{\mathrm{gr}}$ Poncet s'avère explicite à ce sujet, n'hésitant pas à rappeler ses interventions verbales ou à travers ses lettres épiscopales. Le lendemain de l'élection du Lavelua Kapeliele Tufele, un attroupement se forme et le résident doit user d'intimidation pour l'amener à se disperser. $\mathrm{M}^{\mathrm{gr}}$ Poncet écrit :

« Le dimanche suivant 23 avril, ils purent entendre à l'église la lecture d'une lettre de l'évêque stigmatisant, comme il le méritait, le scandale de cette manifestation si irrespectueuse à l'égard du représentant du pouvoir protecteur. » (Poncet, 1972 : 189)

Cette influence sur le temporel a bien sûr ses limites et $\mathrm{M}^{\mathrm{gr}}$ Poncet lui-même explique qu'en 1953 il s'opposa avec succès une première fois à la destitution du Lavelua. Puis une deuxième crise intervint et cette fois-ci les chefs, conscients des recommandations épiscopales, décident de passer outre. $\mathrm{Au}$ lieu de détecter dans cette action un affaiblissement de l'autorité religieuse, ne faut-il pas plutôt conclure à l'apparition d'une maturité politique. À Futuna, la mission reste très puissante et le père Cantala bénéficie d'une grande influence, que l'administration dénomme «immixtion». C'est ainsi que les codes de lois de Sigave et d'Alo ont toujours cours, les remaniements de 1954 et 1960 destinés à les adapter aux réalités nouvelles étant la preuve de leur vivacité.

Les résidents ont souvent critiqué dans leurs rapports la mission ${ }^{21}$, pour la simple raison que son influence morale dépassait leurs compétences administratives. Dans le même temps, lorsque le résident André quitte l'archipel en 1954, il reconnaît que :

« la mission catholique s'est intégrée socialement et économiquement au pays, au point de vivre avec lui dans une sorte de symbiose [...]. Jusqu'ici, il me semble que cette influence bénéfique a été surtout religieuse et morale [...]. À mon sens, il importe que dès maintenant cette influence se fasse sentir également en matière économique de façon plus moderne. $»^{22}$

$\mathrm{M}^{\mathrm{gr}}$ Poncet insiste à plusieurs reprises sur la nécessaire neutralité de son clergé, écrivant même en 1957 :

« Les pères doivent se tenir en dehors des questions politiques ici, mais qu'on doit obéissance au pouvoir établi, et donc qu'on ne peut approuver un mouve-

20. Rapport du résident Folies-Déjardins au gouverneur de la Nouvelle-Calédonie, Wallis, 17 décembre 1951 (archives de la Nouvelle-Calédonie, fonds Wallis-et-Futuna, pièce 25).

21. Rapport du résident Cresson au gouverneur de la Nouvelle-Calédonie, Wallis, 14 septembre 1949. (DWF, carton rapports)

22. Lettre du résident à monseigneur, Mata-Utu, 29 décembre 1954 (archives de Lano, carton DVII, chemise A3). 
ment de rébellion contre ce pouvoir, même si on le juge inapte à gouverner. $»^{23}$

Il délivre même un satisfecit à l'administration coloniale, indiquant dans une note :

« ̀̀ part quelques rares erreurs d'aiguillage, le protectorat a été bien pour les populations de Wallis-et-Futuna ».

\section{La lente agonie d'un protectorat devenu anachronique}

Longtemps, le protectorat avait été théorique et le résident Bécu a même pu dire dans les années 1920 qu'il s'agit d'un " gros mot pour une petite chose ». Avec l'arrivée du résident David en 1933 la situation évolue en faveur de l'administration coloniale. Mais peut-on justement parler de colonisation dans une île où le résident, un chancelier et un opérateur radio formaient l'ossature de la présence française?

À la fin de la Deuième Guerre mondiale, on constate un effacement provisoire de la présence française. Seule la résidence administrative et l'hôpital, frappés de vétusté, subsistent. L'école a arrêté de fonctionner et la résidence considère qu'en 1948 seulement vingt Wallisiens parlent la langue française. Le traitement rapide et efficace d'une épidémie de grippe à Wallis en 1947 redonne à l'administration un certain prestige. La construction de réservoirs à eau est reprise et en 1949 l'impôt de capitation est rétabli. Il n'est plus attribué au gouvernement français mais affecté au budget de Wallis-et-Futuna et il s'élève par homme de 18 à 55 ans à 40 francs à Wallis, et à 100 francs à Futuna. En revanche, le travail forcé, suite à un décret autoritaire controversé du Lavelua, est définitivement aboli par le gouverneur Cournarie en 1950 :

«Les travaux de l'administration, sans exception, seraient à l'avenir payés aux indigènes : tels les travaux $\mathrm{du}$ wharf, de la route, des dispensaires. Seuls les travaux de village pourraient être ordonnés par le chef de village. $»^{24}$

Depuis la guerre, le résident dirige une modeste force de police. Durant la guerre, ce sont des Calédoniens ; puis, en 1947, des Tahitiens ; puis à nouveau un détachement de soldats calédoniens, finalement remplacé en 1952 par deux gendarmes métropolitains accompagnés de quinze auxiliaires calédoniens.

Parallèlement, l'installation de la société Lavoix à la fin 1947 à Wallis sous la direction
d'Emmanuel Brial et l'instauration d'une ligne maritime tous les quatre à six mois avec la Nouvelle-Calédonie permettent le véritable désenclavement de l'île. Ce système est renforcé, en 1949, par la mise en place d'une rotation tri-annuelle comprenant Futuna et, en 1957, par la création d'une ligne aérienne pour les passagers, la reine Aloisia ayant accepté après d'âpres marchandages la cession de l'ancienne piste américaine de Hihifo. L'application du FIDES à Wallis-et-Futuna à partir des années 1950 permet à l'administration, grâce aux subventions annuelles, d'intervenir dans tous les domaines. Des infrastructures commencent alors à sortir de terre. C'est ainsi que se met en place une fonction publique locale, dont les salaires augmentent notablement après la grève de 1951 . Le véritable bouleversement intervient en 1952 quand les mandats des expatriés en Nouvelle-Calédonie ou aux Nouvelles-Hébrides commencent à parvenir dans les familles.

Sur le plan politique, dès 1949, le gouverneur Cournarie demande au résident Cresson un projet de constitution pour Wallis. En 1950, le même gouverneur général prie l'évêque de lui remettre un projet de réforme des assemblées publiques ou fono. En 1951, le statut de Wallis-et-Futuna dans l'Union française est à l'étude, le sujet de l'annexion étant débattu. Or la résidence comme la mission souhaitent au préalable qu'une constitution coutumière soit rédigée. Le résident souhaite que le roi lui fasse parvenir une proposition et ce dernier fait demander à $\mathrm{M}^{\mathrm{gr}}$ Poncet de lui rédiger un tel projet. Ce dernier reprend la constitution wallisienne de 1870 qui représentait « la coutume du moment, quelque peu perfectionnée dans le sens démocratique ». Le préambule insiste sur l'alliance de la coutume et de la religion :

« L'État wallisien met principalement sa confiance dans la protection de Dieu Tout-Puissant. Il professe le respect le plus absolu pour la religion catholique romaine, qui est celle de l'ensemble de la population. » (Poncet, 1972 : 220).

Peu après, le résident informe l'évêque que le monarque lui a fait parvenir un projet, suranné dans ses grandes lignes, qu'il a dû écarter. À partir de ce moment-là, $\mathrm{M}^{\mathrm{gr}}$ Poncet reste à l'écart tout en notant lors de la réunion des missionnaires en 1952 que le jeune clergé autochtone est favorable au maintien du protectorat. Afin d'éviter toute méprise, il autorise ses membres à donner leur sentiment en privé, du moment qu'ils

23. Compte-rendu de la réunion annuelle des missionnaires, Lano, 27 décembre 1957 (archives de Lano, carton DVI, chemise A).

24. Décret $n^{\circ} 9$ du roi des Wallis sur les prestations gratuites, Mata-Utu, 24 février 1950 (archives de Lano, carton DVII, chemise A2. Décision du gouverneur général Cournarie, Mata-Utu, 27 avril 1950). 
n'abordent pas le sujet à l'église. Fin 1953, avant de partir assister à la canonisation de SaintPierre-Chanel programmée pour 1954, il laisse des consignes strictes :

"Changement de statut de W.F. Que les pères ne prennent pas parti : qu'ils ne se mêlent pas des questions politiques proprement dites - soit en prêchant, soit autrement : c'est la règle de l'Église, et c'est le seul moyen d'éviter autant que possible les murmures de certains. Le seul point dangereux : les lois laïques ; il faudrait que les gens réclament à ce sujet ; mais je m'en occupe et je m'en occuperai moi-même. $»^{25}$

En 1953, le sénateur calédonien Henri Lafleur propose l'érection de Wallis-et-Futuna en circonscription électorale rattachée à la NouvelleCalédonie, projet qui est rejeté par le Sénat en 1954. Le but recherché est d'affaiblir le parti calédonien dominant de l'époque, l'Union calédonienne, à forte tendance autonomiste. À Wallis la résidence et la mission refusent l'aspiration de Wallis-et-Futuna par Nouméa, symbole d'une dépendance administrative accrue pour certains et d'un relâchement des mœurs pour d'autres.

En 1958, les Français de Wallis-et-Futuna votent lors du référendum qui établit la constitution de la $v^{\mathrm{e}}$ République. Peu après, le gouverneur Péchoux propose de remplacer le protectorat par le statut de territoire d'Outre-Mer. Le résident Fauché et le délégué de Futuna Camille Gloannec ${ }^{26}$ ont assez d'habileté pour que le Lavelua Kulimoetoke et les deux rois de Futuna fassent discrètement une démarche conjointe auprès du président de la République française. Afin d'être accepté par la mission comme par les chefs coutumiers, ce projet est dérogatoire sur trois points : perpétuation du droit coutumier pour le civil ; maintien du statu quo foncier; conservation d'un enseignement confessionnel (Gata, 2001 : 49). Cet accord est scellé le 5 octobre 1959 lors de la venue du ministre de la France d'Outre-Mer, Jacques Soustelle.

Les deux rois de Futuna sont arrivés le jour même par le navire de guerre le Tiaré et, après que chacun d'eux ait reçu une décoration, le ministre leur explique que le nouveau statut respecterait les coutumes de l'archipel. Quelques Futuniens s'inquiètent de ce statut qu'ils rapprochent de celui de colonie (koloni). En revanche, ce projet bénéficie de l'appui des émigrés wallisiens et futuniens qui se rendent compte des avantages qu'ils tireraient de la nationalité française (statut de résident, considération, allocations familiales). Quant à $\mathrm{M}^{\mathrm{gr}}$ Poncet, il est rassuré par ses entrevues avec le ministre, ses promesses étant confirmées par le gouverneur Péchoux :

«Je comprends parfaitement les inquiétudes que peut nourrir la mission lorsqu'elle redoute que les nouveaux statuts n'apportent l'application dans l'archipel des lois non conformes à celles de l'Église, et non conformes aux désirs des populations [...]. Enfin, des garanties supplémentaires se trouveront données à la mission par le fait que les lois métropolitaines pour être applicables dans l'archipel, devront être promulguées, et que cette promulgation n'interviendra que sur l'avis de l'assemblée locale. » (Poncet, 1972: 225)

Il est prévu que le résident et l'évêque préparent un avant-projet mais finalement le décret est préparé directement à Paris. Le 9 décembre 1959 un Lancaster apporte le texte du décret fixant le référendum au 27 décembre. Les Wallisiens et les Futuniens sont appelés à répondre « oui » ou « non » à la question suivante :

« Désirez vous que les îles Wallis et Futuna fassent partie intégrante de la République française sous la forme d'un territoire d'outre-mer?».

Il prend une tournure unanimiste avec $94,47 \%$ de « oui », avec néanmoins plus de réserve de la part des Futuniens ${ }^{27}$. Parallèlement, une assemblée provisoire est mise en place le 17 février 1960. Elle comporte les trois rois, les principaux chefs des deux archipels et l'évêque. Elle discute le projet de loi, qui est ratifié par le parlement français le 29 juillet 1961, entraînant la création du territoire d'Outre-Mer des îles Wallis-et-Futuna ${ }^{28}$.

25. $\mathrm{M}^{\mathrm{gr}}$ Poncet : Notes, feuilles volantes dans le cahier 1950-1955 (archives de Lano, carton DVII, chemise A4).

26. «Ma mission confirmée et précisée par le résident de France, était comme lui-même l'avait entreprise à Wallis, de convaincre les autorités religieuses, très influentes depuis le massacre du premier missionnaire catholique [...] et, avec leur accord et leur appui, les autorités coutumières et leurs "sujets" de demander à la France l'octroi d'un statut de territoire d'outre-mer » (témoignage de M. Camille Gloannec, interview dactylographié réalisé par Allison Lotti et reproduit dans son DEA, pp. 95-101, ici p. 96).

27. Allison Lotti donne le détail de la rixe du 31 juillet 1960 qui entraîna la mort de plusieurs Futuniens originaires d'Alo : « Il ne s'agissait pas d'un simple combat comme on en voyait régulièrement à la sortie de la messe, à main nues sans armes. Un Futunien trouva un fusil et tira en l'air pour tenter d'arrêter la violence, les deux gendarmes réussirent sans trop de mal à le désarmer, et par la suite le délégué fit procéder à la confiscation de tous les fusils de l'île. Des hommes ne se relevèrent pas, ils furent transportés chez eux et moururent plusieurs jours après » (Lotti, $2005: 46$ ).

28. "Article 2. Les originaires des îles Wallis et Futuna ont la nationalité française. Ils jouissent des droits, prérogatives et libertés attachées à la qualité de citoyen français et sont soumis aux mêmes obligations. Ceux d'entre eux qui n'ont pas le statut de droit commun conservent leur statut personnel tant qu'ils n'y ont pas expressément renoncé. Article 3. La République 
Le dernier résident, M. Fauché, quitte l'archipel le 9 juillet au profit du premier administrateur supérieur qui arrive à Wallis le 3 octobre. Dans le même temps, $\mathrm{M}^{\mathrm{gr}}$ Poncet, conscient des nouveaux enjeux et de son grand âge, demande à Rome d'être relevé de ses fonctions. Son successeur, $\mathbf{M}^{\mathrm{gr}}$ Darmancier, est sacré à Nouméa en avril 1962 et débarque à Wallis en mai. Une page de l'histoire de Wallis-et-Futuna est tournée.

\section{Une période de profondes mutations économiques}

Les deux dernières décennies du protectorat correspondent à une nouvelle orientation sociale et économique de Wallis-et-Futuna, l'exportation de travailleurs remplaçant progressivement l'exportation du coprah.

\section{Un effondrement inattendu des exportations de coprah}

Suite aux rapports des résidents, le ministère de la France d'Outre-Mer propose un premier train de mesures économiques: relance du coprah ; expérimentation de nouvelles cultures commerciales (ananas, arachide, ricin) ; construction d'un hôtel; engagement de la maind'œuvre locale sous contrat; desserte régulière des deux îles.

En 1948, les exportations de coprah reprennent, Futuna procurant 450 tonnes des 473 tonnes expédiées vers la Nouvelle-Calédonie. La même année le commerce local se restructure, la maison Lavoix de Nouméa rachetant les comptoirs locaux et installant comme associés minoritaires les frères Brial. Un nouveau problème apparaît, la détérioration des termes de l'échange, qui se concrétise par l'envol des prix des produits importés. Aussi les Wallisiens, et, dans une moindre mesure les Futuniens, se désintéressent-ils progressivement de cette culture à partir de 1950. Les récoltes d'après-guerre font encore illusion mais l'état de la cocoteraie devient inquiétant. Divers moyens de lutte sont préconisés et un technicien venu d'Indochine insiste sur la nécessité de nettoyer les plantations et leurs abords, peine perdue ! Quant à l'évêque, il note :
«Pauvreté générale par manque de coprah. Certains, peut-être beaucoup, manquant la messe ou l'école faute de mea lafi. ${ }^{29}$

L'administration met en place un séchoir à coprah, mais il semble que sa préparation reste médiocre. Par ailleurs, la population consomme beaucoup de noix et les tabous réguliers sur les cocos verts ne sont que partiellement respectés. Futuna, indemne de l'Oryctes, voit sa production diminuer plus lentement, mais sûrement, du fait de l'absence de tout souci agronomique et d'un système de cueillette. Parallèlement, les Wallisiens récoltent à nouveau le troca à destination de Nouméa, quelques tonnes en 1950, 60 tonnes en 1951.

Le déclin est manifeste après 1951 et l'absence de statistiques pour Wallis s'avère significatif. Les résultats agricoles étant décevants, le résident note en 1951 en se référant uniquement aux standards économiques occidentaux : " la pauvreté générale d'une population qui vit au jour le jour ». Cette détérioration continue en 1953 et le brigadier de gendarmerie constate :

« Il y a 3000 hectares de cocotiers à Wallis mais l'Oryctes les ravage inexorablement et, découragée, la population refuse de continuer le débroussage. Les cultures vivrières subissent de façon beaucoup moins explicable la même désaffection [...]. Si la population est sous-alimentée, personne n'est encore mort de faim. $»^{30}$

Aussi, dès les années 1950, parle-t-on d'un appauvrissement de l'archipel. De fait, le parasitisme des cocoteraies est le facteur déclencheur, mais l'abandon de la seule culture commerciale de l'archipel s'explique surtout par un entretien désormais pénible et par la baisse des cours. Pourtant, la résidence cherche à fixer au plus haut le prix d'achat négocié avec les maisons de commerce calédoniennes tout en fixant une limite à la majoration des prix des biens de consommation par rapport à ce même territoire. C'est ainsi que Wallis, dont la production annuelle s'élève en moyenne avant-guerre à 596 tonnes, tombe à 135 tonnes au cours de la période 1946-1950 et à 53 tonnes pour les années 1956-1959. Futuna, épargnée par l'Oryctes, connaît une régression moins rapide mais régulière, passant de 517 tonnes pour la période

garantit aux populations du territoire des îles Wallis et Futuna le libre exercice de leur religion, ainsi que le respect de leurs croyances et de leurs coutumes en tant qu'elles ne sont pas contraires aux principes généraux du droit et aux dispositions de la présente loi. Elle prend toutes mesures propres à assurer le développement économique du territoire, notamment par l'intermédiaire du fonds d'investissement et de développement économique et social » (loi conférant aux îles Wallis-et-Futuna le statut de territoire d'outre-mer, loi no 61-814 du 29 juillet 1961 publiée au JORF du 30 juillet 1961).

29. $\mathrm{M}^{\mathrm{gr}}$ Poncet, Compte-rendu de la conférence du 16 novembre 1950, Conférences mensuelles 1950-1952, cahier manuscrit (archives de Lano, carton DIV, chemise A5). Mea lafi peut se traduire par « richesses ».

30. Rapport du brigadier de gendarmerie au résident, Mata-Utu, 17 décembre 1953 (archives de l'administration supérieure, Wallis). 
TABLEAU 1. - Estimation et répartition du revenu monétaire en 1961.

\begin{tabular}{|l|c|c|c|c|c|}
\hline Recettes & Coprah & Salaires & Mandats & Autres & Total \\
\hline Millions de F CFP & 2,9 & 5,7 & 4,3 & 2,4 & 15,2 \\
\hline
\end{tabular}

1936-1940, à 344 tonnes pour les trois années 1956-1959. Aussi, la mise en place dès $1959 \mathrm{du}$ nouveau statut entraîne-t-elle de nouveaux flux monétaires, qui sauvent l'économie locale et remettent en cause les équilibres traditionnels.

Le seul capital productif de l'archipel est donc laissé dans un état de semi-abandon et les Wallisiens comme les Futuniens se tournent vers l'émigration.

\section{L'émigration comme réponse à la crise démogra- phique, sociale et économique}

Sur le plan démographique, on peut noter l'impact de la période américaine, qui se concrétise par une plus grande liberté des jeunes gens vis-à-vis de leurs familles et par la naissance d'un certain nombre de métis, surnommés encore aujourd'hui les Amelika. À l'inverse de nombreux pays, l'immédiate après-guerre correspond à une chute de la natalité, liée à la fin de l'âge d'or. Il est vrai que dans le même temps la mortalité régresse notablement. Le service de santé du protectorat fait ainsi état d'un excédent annuel confortable $(2,5 \%)$ qui dure jusque dans les années 1960. Les problèmes posés par cette exubérance démographique sont résolus d'une manière temporaire par une émigration intensive vers les territoires francophones de la Mélanésie du Sud.

En effet, l'accroissement démographique et le désir d'une vie nouvelle poussent de nombreux Wallisiens et Futuniens à s'expatrier. Enfin, il apparaît que, jusqu'en 1960, la majorité des migrants appartient au groupe des jeunes astreints aux plus lourdes tâches. Dès la remise en place de moyens de transport en 1947, certains quittent l'archipel en tant qu'appelés dans l'armée ou engagés sous contrat. L'idée est avancée dès la fin du XIX ${ }^{\mathrm{e}}$ siècle et quelques tentatives ont eu lieu durant la première moitié $\mathrm{du} \mathrm{xx}^{\mathrm{e}}$ siècle : vers Tahiti en 1913 ; vers Nouméa en particulier par la société Hagen en 1920 ; vers la Nouvelle-Calédonie par les autorités américaines en 1943.

Dès 1946, la Société des îles Loyalty, filiale du groupe Ballande, demande l'autorisation de recruter de la main-d'œuvre à Wallis. Le gouverneur de la Nouvelle-Calédonie refuse momentanément car le problème de l'agitation proaméricaine n'est pas encore résolu. Fin 1946, une émigration perlée se met en place, malgré la pru- dence de l'administration, et, dès 1947, le pouvoir coutumier souhaite l'organiser. Le roi Pelenato Fuluhea, soutenu par la résidence, exprime alors trois vœux : une prime de 250 francs « à titre de récompense pour roi et chefs »; la constitution à Nouméa d'un pécule de 200 francs par mois, prélevé sur les salaires et versé lors du retour ; une durée des contrats de trois ans non renouvelable immédiatement. Quant à l'évêque, il écrit bien plus tard :

« On ne pouvait donc songer à contrecarrer ce phénomène de l'émigration des Wallisiens et des Futuniens. Il fallait toutefois veiller à ce qu'elle se fasse avec ordre, de crainte de voir bientôt ces îles manquer de la main-d'œuvre indispensable à la subsistance de ceux qui y resteraient, dont une importante proportion de vieillards, de femmes et d'enfants, les partants étant surtout des jeunes gens et des hommes valides. » (Poncet, 1972 : 193)

De fait, $\mathrm{M}^{\mathrm{gr}}$ Poncet donne pleinement son accord lorsque l'administration lui donne des assurances quant à la création de groupes homogènes et à la possibilité pour les émigrants d'observer régulièrement leur religion.

En 1947, le premier convoi d'engagés pour la Nouvelle-Calédonie compte quatre-vingts-trois personnes, dont quinze femmes et six enfants. Toutes les demandes du roi de Wallis ont été acceptées et le salaire mensuel est fixé à 600 francs, le logement et le couvert étant assuré par les engagistes. Bientôt, de nombreux problèmes apparaissent, des employeurs ne payant pas les émoluments convenus alors que nombre de Wallisiens quittent leurs patrons sans autorisation. C'est pourquoi, une fois que le seuil de deux cents départs soit atteint en 1948, les résidents limitent l'émigration, en arguant du fait que le déséquilibre entre les sexes devient excessif et que les appelés envoyés dix-huit mois en Nouvelle-Calédonie reviennent avec une « mauvaise mentalité ».

Le clergé de Wallis semble avoir eu une saine perception de la situation, puisqu'à la réunion annuelle des missionnaires en $1951 \mathrm{M}^{\mathrm{gr}}$ Poncet note:

«Je rappelle à tous l'évolution actuelle ici, beaucoup de va-et-vient d'ici en Nouvelle-Calédonie, d'où idées nouvelles, désir de plus de liberté chez les indigènes : en ce quoi il y a du bon, mais excès à éviter. Pour empêcher qu'il en résulte de fâcheux inconvénients et pour prévenir la menace possible du communisme un 
TABLEAU 2. - Évolution de l'émigration vers la Nouvelle-Calédonie (d'après Jean-Claude Roux, 1995 : 320 ).

\begin{tabular}{|l|c|c|c|}
\hline Années & Nbre de départs & Nbre de retours & Balance \\
\hline $1947-1950$ & 279 & 180 & +99 \\
\hline $1951-1955$ & 800 & 327 & +473 \\
\hline $1956-1960$ & 1916 & 912 & +1004 \\
\hline $1961-1962$ & 1079 & 657 & +422 \\
\hline Total & 4074 & 2076 & +1998 \\
\hline
\end{tabular}

jour ou l'autre, il faut s'en tenir sérieusement aux recommandations du Saint-Siège, concernant la doctrine sociale de l'Église : travail rétribué convenablement, etc. et ici à Wallis-et-Futuna, suppression totale du travail forcé par l'entremise des chefs en ce qui concerne la mission. »

La mission se considérant comme responsable de la moralité de ses fidèles, elle n'hésite pas à informer l'évêché de Nouméa de ce qu'elle considère être les faiblesses morales ou politiques des partants ${ }^{31}$.

$\mathrm{M}^{\mathrm{gr}}$ Poncet en 1953 constate que l'émigration est à la croisée de deux dangers : "famine et immoralité. Que faire ? $»^{32}$. Il répond à cette interrogation dans les mêmes instructions en proposant d'agir sur les gens, de limiter les départs, d'amener Benjamin Brial à ne plus jouer le rôle de recruteur et de conseiller le résident.

L'administration ayant accepté depuis 1950 la limitation du nombre des appelés aux cinquante souhaités par la chefferie et la mission, un redémarrage de l'émigration a lieu en 1953. Elle favorise désormais l'émigration familiale souhaitée par la mission et les femmes et les enfants sont prioritaires lors des départs. En 1955 le résident informe l'évêque que les deux-tiers au moins des futurs expatriés à destination des NouvellesHébrides doivent être mariés. On exige des Wallisiens un contrat d'engagement, un permis d'embarquement et les départs sont contingentés à dix par navire de passage.

Désormais, les retours deviennent beaucoup moins nombreux que les départs, signe manifeste d'une véritable réussite de cette émigration de travail. Cette main-d'œuvre insulaire se révéle originale : elle est exigeante quant à ses conditions de logement, elle entend obtenir des rations alimentaires conformes à ses goûts, et il faut que ses représentants coutumiers soient consultés pour toutes les modifications d'embauche. De fait, dès 1955, le haut-commissaire de la République accepte qu'un représentant de la reine participe au règlement des différents litiges.
Dans le même temps, la mission, qui y songe depuis 1952, envoie en 1956 auprès de cette communauté estimée à environ mille deux cents personnes le père Sanualio lorsque le début de la construction du barrage de Yaté implique un départ de deux cents nouveaux émigrants. La même année, les Nouvelles-Hébrides reçoivent un aumônier en la personne du père Soane. Il est remplacé dès 1958 par le père Afalaa mais l'habitude est prise, les Nouvelles-Hébrides et la Nouvelle-Calédonie bénéficient, en plus du clergé local, d'un prêtre autochtone. Ce nouvel encadrement a pour effet de multiplier les dons de la communauté expatriée aux paroisses d'origine. C'est ainsi qu'en 1957 les Futuniens de Calédonie offrent un harmonium à l'église de Sigave ou que les Wallisiens et les Futuniens ont déposé seize enveloppes à la cathédrale de Nouméa afin d'acquérir seize statues.

À Wallis-et-Futuna même, des modifications interviennent. En 1957, un état civil « laïque » devient la règle. Toutes les naissances et tous les décès doivent être signalés à l'administration et les nouveaux mariés doivent venir chercher un livret de famille. $\mathrm{M}^{\mathrm{gr}}$ Poncet nous apprend que cette «formalité civile, demandée maintenant à Wallis-et-Futuna en vue de rédiger un livret de mariage pour tous les époux à partir de janvier 1957 (nécessaire à cause des émigrants en Nouvelle-Calédonie) consistera simplement dans l'inscription du mariage sur le registre de l'état-civil, après le mariage à l'église, et sans le questionnaire protocolaire en usage en France (Voulez-vous prendre une telle pour épouse, etc.) ». Puis en 1958 le résident Rougetet précise qu'il exige désormais, en plus de la caution, un certificat médical ainsi qu'un certificat d'hébergement et de logement. De plus, en raison « du chômage et de la misère qui ont commencé pour les Wallisiens en Nouvelle-Calédonie $»^{33}$, il limite les départs.

Parallèlement, des demandes des Établissements français de l'Océanie pour les chantiers de

31. Lettre de $M^{g r}$ Poncet à $M^{g r}$ Bresson, Lano, 8 avril 1951 (archives de Lano, carton DVII, chemise A2).

32. $\mathrm{M}^{\mathrm{gr}}$ Poncet, Notes, feuilles volantes dans le cahier 1950-1955 (archives de Lano, carton DIV, chemise A4).

33. Liste des passagers pour Nouméa par avion et par bateau, par le résident de France, le médecin-capitaine Rougetet, Wallis, 15 avril 1958 (archives de Lano, carton DVII, chemise A3). 
Papeete sont rejetées alors que des propositions émanant de la résidence de France aux Nouvelles-Hébrides sont acceptées. Le résident considère en effet que, sur les plantations, les recrutés «trouveront un milieu agricole analogue aux Wallis $»^{34}$. Ce sont cent cinquante-huit personnes qui se rendent ainsi à Port-Vila en 1952 et si l'on en croit le père O'Reilly, les départs suivants se font à la cadence de cinq à six par mois, par relations directes. Ils y sont rapidement appréciés pour leur capacité à dépasser les quotas hebdomadaires, pour leur propreté, leur pratique religieuse régulière ou encore leur sobriété. En ce qui concerne leurs rapports avec les colons, un inspecteur du travail note le « caractère fier et ombrageux de ces travailleurs qui se considèrent comme supérieurs $»^{35}$. Cette caractéristique a pour le patronat des incidences positives car il renforce l'élasticité de l'emploi alors que les Wallisiens et les Futuniens, de par leur redoutable force de travail et de par leur encadrement par des représentants coutumiers et le clergé catholique, obligent les employés originaires des autres communautés à un travail plus assidu. Comme le note Jean-Claude Roux, les Wallisiens et Futuniens vont constituer, à terme, dans le Condominium aussi bien qu'en Nouvelle-Calédonie « des communautés bien soudées, individualisées et protégées qui vont devenir lentement partie prenante de la vie politique locale et être souvent mal supportées voire rejetées par les tenants des autonomismes locaux qui voient en eux des auxiliaires aux politiques administratives de la métropole » (Roux, 1991 : 615).

Ces migrations sont régulièrement contrariées par le statut des Wallisiens et des Futuniens, originaires d'un " pays » protégé par la France ${ }^{36}$, ne possédant ni les avantages d'un État souverain, ni les facilités d'un territoire membre de l'Union Française. Aussi le changement institutionnel de 1961 libéralise-t-il les conditions de migration, à l'aube d'une nouvelle époque.

\section{Conclusion}

De 1942 à 1961, Wallis-et-Futuna a donc vécu une période de transition, quittant les sentiers connus pour entrer à petits pas dans le monde moderne. La mission, devenue évêché en 1936, a commencé sa mutation, aidée en cela par le rôle accru du clergé autochtone. Les trois chefferies symbolisent une coutume qui reste omniprésente et multiforme. Le pouvoir des commerçants exportateurs-importateurs persiste, mais il se fait plus discret. Quant à la résidence, elle vit alors son âge d'or, du fait de l'effacement progressif des autres pouvoirs traditionnels, alors qu'il n'existe pas encore de contre-pouvoir politique.

Mais la grande nouveauté, initiée dès le temps des Amelika, reste l'entrée de Wallis-et-Futuna dans le circuit des migrations. En effet, l'apparition de nouveaux besoins, l'effondrement de la mono-production locale de coprah et une surpopulation avérée, poussent les Wallisiens et Futuniens à émigrer.

Un parti des migrants se forme peu à peu durant les dix-neuf dernières années du protectorat et il explique ou, du moins, il facilite le changement radical de statut définitivement adopté en 1961. En effet, si le changement concret de statut fut bien initié après l'arrivée du général de Gaulle à la présidence de la République en mai 1958, divers projets avaient déjà vu le jour depuis 1949. Après soixante-quatorze ans de protectorat, Wallis et Futuna devient partie intégrante de l'ensemble français tout en bénéficiant dans les faits d'une large autonomie.

\section{BIBLIOGRAPHIE}

Aldrich Robert, 1993. France and the South Pacific since 1940, University of Hawaii press, $413 \mathrm{p}$.

ANGLEviel Frédéric, 1997. Wallis and Futuna. 19451953. The post World War II crisis and reconstruction, in Aldrich Robert and Isabelle Merle (eds), France Abroad. Indochine, New Caledonia, Wallis and Futuna, Mayotte, Sydney, University of Sydney, pp. 47-78.

—, 2003. Église et éducation à Wallis-et-Futuna, 1837-1961. De la formation des cadres religieux à l'encadrement de toute la jeunesse, Mélanges offerts à Gérard Cholvy, Montpellier, université de Montpellier III, pp. 503-518.

ANONYME [GarRity J.-J. ?], 1944. Notes on Wallis Island, rapport dactylographié, collection personnelle (don du père Claude Rozier).

34. Télégramme du résident au gouverneur Angamarre, Wallis, $1^{\mathrm{er}}$ novembre 1951 (DWF, carton correspondance).

35. Télégramme du résident de Wallis-et-Futuna au gouverneur Angamarre, Wallis, $1^{\mathrm{er}}$ novembre 1951. (DWF, carton correspondance). Il est à noter que ce gouverneur avait été en poste comme fonctionnaire aux Nouvelles-Hébrides dans les années 1920.

36. Lettre du résident de France à monsieur le commissaire général de la République dans l'Océan Pacifique, Mata-Utu, $1^{\mathrm{er}}$ juin 1954 (DWF, carton Archives divers). « Le problème sera plus compliqué aux Nouvelles-Hébrides tant que l'émission des mandats postaux pour Wallis y sera interdite, mais déjà les employeurs règlent le transport de leur futur employé au commandant du Maria Del Mar, au moment où s'effectue le débarquement à Port-Vila ». 
Charbonnier Robert $\left(\mathrm{D}^{\mathrm{r}}\right)$, 1980. Chroniques d'un séjour mouvementé à Wallis-et-Futuna dans le Pacifique en guerre, Bruxelles, Presses universitaires de Bruxelles.

Gata Petelo, 2001. Du protectorat au statut de Territoire d'outre-mer, in Huffer Élise et Leleivai Petelo (eds), 2001. Futuna. Mo Ona Puleaga Sau (aux deux royaumes), Institute of Pacific Studies, 188 p., pp. 43-50.

Huffer Élise et Petelo Leleivai (eds), 2001. Futuna. Mo Ona Puleaga Sau (aux deux royaumes), Institute of Pacific Studies, 188 p.

Likuvalu Apeleto, 1975. L'école missionnaire aux îles Wallis-et-Futuna, mémoire de licence des sciences de l'éducation, université de Lyon II, 49 p.

LotTi Allison, 2005. Le passage du statut de Protectorat à celui de Territoire d'outre-mer à Wallis-etFutuna (1958-1962), DEA d'histoire, université de Bordeaux III, $152 \mathrm{p}$.

Moyse-FAurie Claire et Angleviel Frédéric, 2002. Futuna ou « l'enfant perdu »... Un timide biculturalisme, Hermès 32-33 : La France et les outre-mers. L'enjeu multiculturel, pp. 377-384.

PANOFF Michel, 1963. Situation présente de la société futunienne, Journal de la Société des Océanistes 19, pp. 149-156.

Pechberty Dominique, 2004. Les Uvéens en Nouvelle-Calédonie, The Journal of Pacific Studies 27-1, pp. 63-81.
—, 2004. Vivre la coutume à G9]Uvea (Wallis), Paris, L'Harmattan, Mondes océaniens, 325 p.

Poncet Alexandre (Mgr), 1972. Histoire de l'île Wallis. Le protectorat français, Paris, Publications de la Société des Océanistes 23, 234 p.

Regnault Jean-Marc, 2004. La France Libre, Vichy et les Américains: des relations difficiles dans le Pacifique en guerre. L'exemple des îles Wallis-etFutuna (1940-1942), Revue Outre-Mers 344-345, pp. 181-200.

Roux Jean-Claude, 1987. Pouvoir religieux et pouvoir politique à Wallis-et-Futuna, in Paul De Deckker et Pierre Lagayette (éds), États et pouvoirs dans les territoires français du Pacifique, Paris, L'Harmattan, pp. 54-80.

_, 1991. Espaces coloniaux et société polynésienne de Wallis-et-Futuna (Pacifique central), vol. 1 ; Chronique du quotidien colonial; vol. 2 : Un archipel en mutation par translation géographique en Mélanésie $d u$ Sud, thèse de doctorat d'État, université de Paris I, 526 p. et 445 p.

—, 1995. Wallis-et-Futuna. Espaces et temps recomposés. Chroniques d'une micro insularité, Bordeaux, université de Bordeaux III, CRET, Îles et archipels 21, $404 \mathrm{p}$. 